\title{
High Prevalence of Low-Level Parasitemia With Plasmodium vivax in Makira-Ulawa Province Presents a Challenge for the Diagnosis and Eradication of Malaria in Solomon Islands
}

\author{
James Fink, MBBS, MPH, FACP, FRACP, Peter D. Jones, MBBS, PhD, DCH, FRACP, GAICD \\ Department of Health Sciences and Medicine, Bond University, Robina, Gold Coast, Queensland, Australia
}

\begin{abstract}
Background: Malaria remains endemic in Solomon Islands, but data on malaria in the provinces of Solomon Islands are limited. This study from Makira-Ulawa Province aimed to identify the most prevalent strain of malaria and assess if the available rapid diagnostic test (RDT) was effective in Kirakira Hospital.

Methods: Forty-five patients who presented to Kirakira Hospital with symptoms of fever had a positive malaria parasite smear during a 4-week period in 2017. The parasite count for each smear was calculated. Simultaneous testing using the CareStart Malaria HRP2/pLDH (Pf/pan) Combo RDT was conducted. The data for all malaria parasite smears performed in Makira-Ulawa Province in 2016 were collated for comparison.

Results: All 45 patients diagnosed with malaria in a 4-week period in 2017 were positive for Plasmodium vivax. The median parasite load was 280 parasites per $\mu \mathrm{L}$ (range, 160 to 640 parasites per $\mu \mathrm{L}$ ). None of the 45 CareStart RDTs performed was positive. In 2016 , 5,505 of 17,195 patients (32.0\%) screened had malaria parasites detected on a malaria parasite smear. $P$ vivax was detected in 5,212 (94.7\%) and Plasmodium falciparum in 285 (5.2\%) of patients with malaria.

Conclusion: $P$ vivax is the predominant strain of malaria present in Makira-Ulawa Province. RDTs were not helpful in the diagnosis of malaria at Kirakira Hospital. The parasite load detected in the 45 patients diagnosed with malaria in this study was low. A focus on attempting to eradicate $P$ vivax in the community through improved compliance with treatment protocols is suggested as a possible way forward to best manage malaria in Makira-Ulawa Province.
\end{abstract}

Keywords: Artemisinins, malaria, malaria-falciparum, malaria-vivax, Melanesia, point-of-care testing, primaquine

Address correspondence to Peter D. Jones, MBBS, PhD, DCH, FRACP, GAICD, Department of Health Sciences and Medicine, Bond University, 1 University Dr., Robina, Gold Coast, Queensland, 4229, Australia. Tel: +61 75595 5205. Email: pejones@bond.edu.au

\section{INTRODUCTION}

Malaria remains endemic in Solomon Islands, but progress toward eradication has been slow and challenging. Despite a reported reduction of $89 \%$ in confirmed malaria cases from 1993 to $2011,{ }^{1}$ wide discrepancies exist in the reported incidences across the 9 provinces of Solomon Islands. In 2011, rates ranged from 1.6 cases per 1,000 population in Isabel Province to 96.8 cases per 1,000 population in Guadalcanal Province. ${ }^{2}$

Since 2009, the diagnosis and treatment of malaria in Solomon Islands has been available free of charge through public health facilities. Artemisinin-based combination therapy $(A C T)$ has been the first-line treatment for all febrile cases of acute malaria, with 14 days of primaquine used for radical treatment of Plasmodium vivax. Glucose-6phosphate dehydrogenase (G6PD) screening has been a requirement prior to commencing primaquine treatment. ${ }^{3}$
Limited data are available about malaria in MakiraUlawa Province of Solomon Islands. Makira-Ulawa is the fifth largest province by population, estimated at 52,000 inhabitants in 2017, and is located approximately $180 \mathrm{~km}$ southeast of Honiara, the capital city of Solomon Islands. ${ }^{4}$ Makira-Ulawa has one small hospital located in the provincial capital Kirakira. Trained microscopists perform as many as 50 malaria parasite smears each day at Kirakira Hospital. The hospital also has a store of rapid diagnostic test (RDT) cartridges that have been shown to be accurate in diagnosing acute malaria cases in other settings. However, the nurses at Kirakira Hospital had stopped using the RDT cartridges because they were "never positive" and "did not work." No specific data were available on when the nurses stopped using the RDTs, but they had not been observed using them since Bond University medical students started completing placements in Kirakira Hospital in 2013. 
In response to these practical observations, we performed a small prospective study of patients presenting to Kirakira Hospital with fever and a positive malaria parasite smear during a 4-week period in 2017. Each of these patients also underwent an RDT cartridge test. In addition, we reviewed the laboratory results for all malaria parasite smears performed during 2016 in Makira-Ulawa Province.

\section{Information About Malaria}

Malaria is a mosquito-borne infectious disease that, according to the World Health Organization (WHO), is responsible for more than 200 million episodes of illness per year across the globe, resulting in up to 400,000 deaths annually. Malaria is caused by the transmission of a parasite from the female Anopheles mosquito into humans. The disease has 4 known strains, but 2 strains-Plasmodium falciparum and $P$ vivax -are responsible for the majority of clinical disease globally. Malaria disproportionately affects the poorest countries of the world, with $85 \%$ of the total disease burden borne by Africa and India. ${ }^{5}$ United Nations Sustainable Development Goal 3.3 is to end the epidemics of acquired immunodeficiency syndrome, tuberculosis, and malaria by $2030 .^{6,7}$ Attaining this goal for malaria is looking increasingly out of reach for the Pacific countries.

$P$ falciparum is the primary strain responsible for severe malaria in Africa and India and accounts for more than $90 \%$ of cases of malaria globally. $P$ vivax is principally seen in Southeast Asia, South America, and the Western Pacific. Although the acute illness associated with $P$ vivax malaria is less severe than the illness associated with $P$ falciparum, $P$ vivax has the potential to cause recurrent infection because it can lie dormant in the liver. Chronic infection with $P$ vivax malaria can cause anemia and splenomegaly, can adversely affect pregnancy, and is a significant cause of morbidity and mortality in Solomon Islands.

Since 2001, the WHO has recommended the use of ACT for acute treatment of malaria because of its efficacy against both the $P$ falciparum and $P$ vivax strains. ${ }^{8}$ Since the introduction of ACT, the number of malaria cases has been reduced in the Southwestern Pacific. ACT was made the first-line treatment for acute malaria in Solomon Islands in 2009 , and during the next 6 years to 2015, the number of malaria cases in Solomon Islands dropped by $75 \%$.

From 2015 to 2020, however, the rate of decline in the number of malaria cases slowed in Solomon Islands. The 2019 malaria report from the WHO identifies $P$ falciparum as responsible for more than $60 \%$ of malaria in Solomon Islands. ${ }^{5}$ However, evidence suggests that $P$ vivax is now the overwhelming predominate strain of malaria in the provinces. $^{9}$ Actual data from the remote provinces of Solomon Islands are very limited; the WHO figures are calculated estimates. Research from Kirakira has shown that these estimates can be very different from actual clinical data taken from the medical records in Solomon Islands. ${ }^{10}$

\section{METHODS}

Ethics approval to conduct the research was granted by the Human Research Ethics Committee of Bond University (BURO 15397).

Patients presenting to the outpatient department of Kirakira Hospital with a fever during April 2017 had a malaria parasite smear taken and assessed by microscopists who
Table 1. Malaria Parasite Smear (MPS) Results for Febrile Patients Presenting to Kirakira Hospital in April 2017

\begin{tabular}{lccc}
\hline $\begin{array}{l}\text { MPS } \\
\text { Positive }\end{array}$ & $\begin{array}{c}\text { Malaria } \\
\text { Species }\end{array}$ & $\begin{array}{c}\text { Parasites } \\
\text { Observed per 200 } \\
\text { White Blood Cells }\end{array}$ & $\begin{array}{c}\text { Calculated } \\
\text { Parasite Load }\end{array}$ \\
\hline 45 & $\begin{array}{c}\text { Plasmodium } \\
\text { vivax }\end{array}$ & $\begin{array}{l}\text { Median: } 7 / 200 \\
\text { Range: 4-16/200 }\end{array}$ & $\begin{array}{l}\text { Median: } 280 / \mu \mathrm{L} \\
\text { Range: } 160-640 / \mu \mathrm{L}\end{array}$ \\
\hline
\end{tabular}

Note: One parasite per 200 white blood cells is calculated as being the equivalent of 40 parasites per $\mu \mathrm{L}$.

identified the strain of malaria and recorded the number of parasites per 200 white blood cells. The number of parasites per $\mu \mathrm{L}$ is estimated by assuming an average count of 8,000 white blood cells per $\mu \mathrm{L}$, so 1 parasite per 200 white blood cells is equivalent to 40 parasites per $\mu \mathrm{L}$. A count of $<1,000$ parasites per $\mu \mathrm{L}$ is considered a low level of parasitemia in acute malaria.

Consent was obtained from patients with a positive malaria parasite smear to have an RDT performed on their blood sample. ${ }^{11,12}$ RDTs were only performed on patients with a positive malaria smear.

The available RDT at Kirakira Hospital was the CareStart Malaria HRP2/pLDH (Pf/pan) Combo. The CareStart RDT detects the histidine-rich protein 2 antigen of $P$ falciparum and Plasmodium lactate dehydrogenase that is produced by all 4 species of malaria. ${ }^{13}$

The microscopists at Kirakira Hospital evaluate malaria parasite smears for the entire province. Outside of Kirakira Hospital, nurses at nurse-run health clinics assess patients for fever and send malaria parasite smears to the hospital. We audited all of the malaria parasite smears evaluated at Kirakira Hospital in 2016. The only information available from the audit was the strain of malaria. No information about the parasite load could be retrieved.

\section{RESULTS}

In April 2017, 45 patients who presented with a fever to Kirakira Hospital had a positive malaria parasite smear. All patients were found to have $P$ vivax. The median parasite load was 7 per 200 white blood cells or 280 parasites per $\mu \mathrm{L}$. The highest parasite load detected was 16 per 200 white blood cells or 640 parasites per $\mu \mathrm{L}$ (Table 1). In contrast, none of the 45 CareStart RDTs performed was positive. No patient had a clinically severe case of malaria. All patients were managed as outpatients, and all patients recovered.

In 2016, 17,195 malaria parasite smears were evaluated at Kirakira Hospital, resulting in 5,212 positives for $P$ vivax, 285 positives for $P$ falciparum, and 8 mixed species positives (Table 2). These results show that in 2016, 32.0\% of febrile patients seen in outpatient settings in Makira-Ulawa Province had positive malaria parasite smears and that $P$ vivax was detected in $94.7 \%$ of cases, demonstrating that $P$ falciparum is an uncommon strain of malaria detected in Makira-Ulawa Province.

\section{DISCUSSION}

Our study confirmed the suspicion of the local nursing staff that the RDTs were not effective for diagnosing malaria in Kirakira. This result is at odds with the reported sensitivities of RDTs that range between $75 \%$ and $90 \%$. However, 
Table 2. Malaria Parasite Smear (MPS) Results From the Kirakira Hospital Malaria Laboratory in 2016

\begin{tabular}{|c|c|c|c|c|}
\hline Test/Results & Adults & Infants & Total & Percentage \\
\hline MPS Performed & 16,291 & 904 & 17,195 & $\mathrm{~N} / \mathrm{A}$ \\
\hline MPS Positive & 5,284 & 221 & 5,505 & $32.0^{\mathrm{a}}$ \\
\hline Plasmodium vivax & 5,003 & 209 & 5,212 & $94.7^{b}$ \\
\hline Plasmodium falciparum & 273 & 12 & 285 & $5.2^{\mathrm{b}}$ \\
\hline Mixed species & 8 & 0 & 8 & $0.1^{\mathrm{b}}$ \\
\hline
\end{tabular}

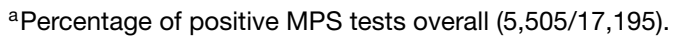

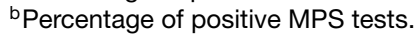

the studies of RDT sensitivities have principally been conducted in the sub-Saharan region of Africa where $P$ falciparum is the main species, the average parasite loads are usually much greater than 1,000 parasites per $\mu \mathrm{L}$, and levels can be as high as 30,000 parasites per $\mu \mathrm{L} .{ }^{14}$ The WHO recommends the use of quality RDTs as standard diagnostic tools for routine malaria case management, but evidence is insufficient to assess if RDTs are useful in low-transmission settings where submicroscopic infections, often not associated with febrile illness, have been shown to have efficient gametocyte production that serves as an ongoing reservoir of potential transmission. ${ }^{15}$ Further research into the sensitivity of the RDTs confirms that a parasite load of $>500$ parasites per $\mu \mathrm{L}$ is often required to trigger a positive result, so RDTs are frequently unable to detect malaria in patients with low parasite loads. In our study, 4 patients had parasite loads $>500$ parasites per $\mu \mathrm{L}$, but all had negative RDT results.

The failure of the RDTs in this study could have several explanations. The kits may have been defective, the staff may not have used them correctly, and the low parasite loads may have contributed to the ineffectiveness of the RDTs. Because we only performed RDTs on patients with positive malaria parasite smears, we could not confirm a falsepositive rate for this particular RDT that has been noted by Swiss researchers conducting fieldwork in Eswatini, formerly known as Swaziland. ${ }^{16}$ RDTs are an important tool for the detection of malaria in resource-poor communities without access to microscopy; however, we had no success with the RDT that was available. Kirakira Hospital had no access to other RDTs that can distinguish between $P$ falciparum and $P$ vivax strains and potentially might have been more effective than the CareStart Malaria HRP2/pLDH (Pf/pan) Combo RDT. ${ }^{17}$ The total budget for all health care in Makira-Ulawa Province is only $\$ 1.3$ million US dollars per annum or the equivalent of \$26 US dollars per annum per capita, so health care resources are extremely limited. ${ }^{18}$

Our study results demonstrate what appears to be a dramatic change in the epidemiology of malaria in Makira-Ulawa Province. $P$ falciparum in this audit was rare and accounted for only $5.2 \%$ of identified cases vs the WHO estimate of approximately $60 \% .^{5} \mathrm{~A}$ possible reason for this change is the introduction of ACT protocols that are highly effective against $P$ falciparum and have been first-line treatment for all cases of malaria in Solomon Islands since 2009. The staff in the Kirakira Hospital malaria laboratory provided anecdotal evidence that the clinics where $P$ falciparum was detected were remote and did not have access to ACT. The introduction of ACT in 2009 proved hugely successful in eradicating $P$ falciparum in Solomon Islands. In Makira-Ulawa Province, however, the situation with $P$ vivax is quite different.

$P$ vivax malaria requires treatment with primaquine for 2 weeks to prevent recurrent infection. However, primaquine can cause acute hemolytic anemia in patients with G6PD deficiency. In Solomon Islands, research indicates that approximately $7 \%$ of the population has a deficiency of G6PD and would be at risk of developing a severe hemolytic reaction when exposed to primaquine. ${ }^{19}$ Consequently, since 2009, the Ministry of Health for Solomon Islands has appropriately recommended that all patients with $P$ vivax malaria be screened for G6PD deficiency, and if safe, to proceed with primaquine treatment. ${ }^{20}$

Implementing this treatment protocol across MakiraUlawa Province has been challenging on many levels. First, health literacy in the community is low, and the need for primaquine treatment when the patient has recovered from the acute febrile episode is often poorly understood by the nursing staff and the patients. Second, adherence with outpatient medication is a particularly challenging problem in Solomon Islands. One strategy to overcome lack of adherence would be to directly observe patients taking their primaquine medication each day to ensure compliance, but resources to implement such a policy are limited. Third, the G6PD screening test is not available at all health centers in Makira-Ulawa Province, and the community and health workforce do not have a clear understanding of why the test is important. ${ }^{21}$

An important issue raised by the study data is how the diagnosis of acute malaria is made. The presence of a fever and a positive malaria parasite smear have been considered sufficient to establish a diagnosis of malaria. What we cannot extract from this audit is whether the low-level parasitemia was the cause of the fever or if patients had other common causes of fever such as acute viral infection. The patients diagnosed with malaria in this study were not sick enough to require inpatient treatment and were effectively managed on an outpatient basis. The highest parasite load among the 45 patients in this study was 640 parasites per $\mu \mathrm{L}$ (16 parasites per 200 white blood cells). This number is below the level likely to cause symptoms of severe malaria (defined as $>1,000$ parasites per $\mu \mathrm{L}$ or $>25$ parasites per 200 white blood cells) and explains why all patients could be successfully managed as outpatients.

Whether the low-level parasitemia identified in this study was sign of a chronic underlying illness and not the cause of the fever is a vexing question because no agreed-upon levels of parasitemia that indicate a definite diagnosis have been established. ${ }^{22}$ Further, what constitutes symptomatic vs asymptomatic malaria has not been firmly established. Chronic or recurrent malaria has long been recognized to cause long-term health problems such as chronic anemia and splenomegaly. ${ }^{23}$

To answer the question of whether the low-level parasitemia identified in this study is a sign of a chronic underlying illness and not the cause of the fever would require screening the population to determine the prevalence of low-grade parasitemia, anemia, and clinical splenomegaly in asymptomatic patients. Such population screening is not part of the current national strategy to address malaria in Solomon Islands. ${ }^{11}$ The number of cases of malaria detected 
in the province would have likely been higher if polymerase chain reaction (PCR) had been used to screen for malaria because this technology is more sensitive in identifying malaria than microscopy or RDT. ${ }^{24}$ Asymptomatic malaria is being recognized with increasing frequency in Southeast Asia, with research using PCR technology revealing that 3 to 4 times as many patients with malaria may be detected with PCR screening alone.

Developing an implementable combination of diagnostic testing and treatment to try to eradicate malaria from Makira-Ulawa Province will be an ongoing challenge. ${ }^{25}$ Malaria is a complex infection; the challenge is to understand the nature of the illness and how that nature might change as the epidemiology changes. Therefore, developing a better understanding of low parasite level and asymptomatic disease is necessary for eradication programs to be successful.

\section{CONCLUSION}

The epidemiology of malaria in Makira-Ulawa Province has changed, with $P$ vivax representing $94.7 \%$ of diagnosed cases and $P$ falciparum having been almost eradicated from the province. To meet the goal of Makira-Ulawa being a malaria-free province by 2030 , population screening is needed to determine the prevalence of low-level parasitemia in the province and if a significant burden of chronic asymptomatic $P$ vivax infection exists. Such a study should be followed by a coordinated public health and implementation campaign that educates the public and health workforce about the importance of adherence to treatment strategies for the eradication of $P$ vivax that include screening for G6PD deficiency and a 14-day course of primaquine.

\section{ACKNOWLEDGMENTS}

The authors are grateful to the 4 senior medical students and local medical officer who helped collect the data while on placement in Kirakira in June 2017. The authors have no financial or proprietary interest in the subject matter of this article.

\section{REFERENCES}

1. Oloifana-Polosovai $\mathrm{H}$, Gwala J, Harrington $\mathrm{H}$, et al. A marked decline in the incidence of malaria in a remote region of Malaita, Solomon Islands, 2008 to 2013. Western Pac Surveill Response J. 2014;5(3):30-39.

2. Progress in malaria control and moving towards elimination in Solomon Islands and Vanuatu. World Health Organization Western Pacific Region. Accessed May 27, 2020. apps.who. int/iris/bitstream/handle/10665/207699/Malaria_control_ SLB_VUT_eng.pdf? sequence $=1$ \&isAllowed $=y$

3. Solomon Islands. World Malaria Report 2018. World Health Organization. Accessed May 27, 2020. www.who.int/malaria/ publications/country-profiles/profile_slb_en.pdf?ua $=1$

4. Provincial profile of the 2009 population and housing census: Makira-Ulawa. Solomon Islands Government. Accessed November 15, 2017. www.statistics.gov.sb/statistics/ demographic-statistics/census

5. World malaria report 2019. World Health Organization. Accessed May 18, 2020. www.who.int/publications-detail/ world-malaria-report-2019

6. Take action for the sustainable development goals. United Nations. Accessed May 18, 2020. www.un.org/ sustainabledevelopment/sustainable-development-goals/
7. Achieving the malaria MDG target: reversing the incidence of malaria 2000-2015. World Health Organization and United Nations Children's Fund. September 2015. Accessed May 18, 2020. www.unicef.org/publications/index_85529.html

8. Mutabingwa TK. Artemisinin-based combination therapies (ACTs): the best hope for malaria treatment but inaccessible to the needy! Acta Trop. 2005;95(3):305-315. doi: 10.1016/j.actatropica.2005.06.009

9. Waltmann A, Darcy AW, Harris I, et al. High rates of asymptomatic, sub-microscopic Plasmodium vivax infection and disappearing Plasmodium falciparum malaria in an area of low transmission in Solomon Islands. PLoS Negl Trop Dis. 2015;9(5):e0003758. doi: 10.1371/journal.pntd.0003758

10. Jones PD, Balasundaram N, D'Costa L, Kacker K, Kaludewa A, Fink J. High perinatal mortality rates persist in Kirakira: the sustainable development goals for health remain out of reach in the provinces of Solomon Islands. J Paediatr Child Health. 2018;54(8):895-899. doi: 10.1111/jpc.13919

11. Xiaodong S, Tambo E, Chun W. Diagnostic performance of CareStart ${ }^{\mathrm{TM}}$ malaria HRP2/pLDH (Pf/pan) combo test versus standard microscopy on falciparum and vivax malaria between the China-Myanmar endemic borders. Malar J. 2013;12:6. doi: 10.1186/1475-2875-12-6

12. Baird JK, Valecha N, Duparc S, White SJ, Price RN. Diagnosis and treatment of Plasmodium vivax malaria. Am J Trop Med Hyg. 2016;95(6 Suppl):35-51. doi: 10.4269/ajtmh.16-0171

13. Cheng Q, Cunningham J, Gatton ML. Systematic review of sub-microscopic $P$. vivax infections: prevalence and determining factors. PLoS Negl Trop Dis. 2015;9(1):e3413. doi: 10.1371/journal.pntd.0003413

14. Berhane A, Russom M, Bahta I, Hagos F, Ghirmai M, Uqubay S. Rapid diagnostic tests failing to detect Palsmodium falciparum infections in Eritrea: an investigation of reported false negative RDT results. Malar J. 2017;16(1):105. doi: 10.1186/s12936-017-1752-9

15. Abba K, Kirkham AJ, Olliaro PL, Deeks JJ, Donegan S, Garner P, Takwoingi Y. Rapid diagnostic tests for diagnosing uncomplicated non-falciparum or Plasmodium vivax malaria in endemic countries. Cochrane Database Syst Rev. 2014;2014(12):CD011431. doi: 10.1002/14651858.CD011431

16. Ranadive N, Kunene S, Darteh S, et al. Limitations of rapid diagnostic testing in patients with suspected malaria: a diagnostic accuracy evaluation from Swaziland, a low-endemicity country aiming for malaria elimination. Clin Infect Dis. 2017;64(9):1221-1227. doi: 10.1093/cid/cix131

17. Global Malaria Programme. Recommended selection criteria for procurement of malaria rapid diagnostic tests. World Health Organization. 2018. Accessed May 27, 2020. apps.who. int/iris/bitstream/handle/10665/259870/WHO-CDS-GMP-2018. 01-eng.pdf?sequence $=21$

18. Solomon Islands Budget 2017 Budget Strategy and Outlook. Accessed February 17, 2021. www.solomonchamber.com.sb/ media/1308/budget-strategy-outlook-vol1.pdf

19. Howes RE, Piel FB, Patil AP, et al. G6PD deficiency prevalence and estimates of affected populations in malaria endemic countries: a geostatistical model-based map. PLoS Med. 2012;9(11):e1001339. doi: 10.1371/journal.pmed.1001339

20. Global Malaria Programme. Testing for G6PD deficiency for safe use of primaquine in radical cure of $P$. vivax and $P$. ovale malaria. Policy brief. World Health Organization. 2016. Accessed March 9, 2018. apps.who.int/iris/bitstream/handle/ 10665/250297/WHO-HTM-GMP-2016.9-eng.pdf;jsessionid= 063C3893ECF2FB0F65EA14CAFE5DBFB3? sequence $=1$

21. Kuwahata $M$, Wijesinghe $R, H o M F$, et al. Population screening for glucose-6-phosphate dehydrogenase deficiencies in Isabel 
Province, Solomon Islands, using a modified enzyme assay on filter paper dried bloodspots. Malar J. 2010;9:223.

doi: 10.1186/1475-2875-9-223

22. Laishram DD, Sutton PL, Nanda N, et al. The complexities of malaria manifestations with a focus on asymptomatic malaria. Malar J. 2012;11:29. doi: 10.1186/1475-2875-11-29

23. Pava Z, Burdam FH, Handayuni I, et al. Submicroscopic and asymptomatic Plasmodium parasitaemia associated with significant risk of anaemia in Papua, Indonesia. PLoS One. 2016;11(10):e0165340. doi: 10.1371/journal.pone.0165340
24. Moreira CM, Abo-Shehada M, Price RN, Drakeley CJ. A systematic review of sub-microscopic Plasmodium vivax infection. Malar J. 2015;14:360. doi: 10.1186/s12936-015-0884-z

25. Imwong M, Nguyen TN, Tripura R, et al. The epidemiology of subclinical malaria infections in South-East Asia: finding from cross-sectional surveys in Thailand-Myanmar border areas, Cambodia, and Vietnam. Malar J. 2015;14:381. doi: 10.1186/s12936-015-0906-x

This article meets the Accreditation Council for Graduate Medical Education and the American Board of Medical Specialties Maintenance of Certification competencies for Patient Care, Medical Knowledge, and Practice-Based Learning and Improvement.

C2021 by the author(s); licensee Ochsner Journal, Ochsner Clinic Foundation, New Orleans, LA. This article is an open (i) access article distributed under the terms and conditions of the Creative Commons Attribution (CC BY) license (creativecommons.org/licenses/by/4.0/legalcode) that permits unrestricted use, distribution, and reproduction in any medium, provided the original author(s) and source are credited. 